\title{
CONTRÔLE DE COHÉRENCE ACTES-DISPOSITIFS MÉDICAUX
}

\author{
Slimane Toubal, Adrien Poreaux, Mathieu Morell, Philippe Mailhe, Jérôme Perrey, \\ Pierre Aubas, Grégoire Mercier
}

ESKA | «Journal de gestion et d'économie médicales »

2018/2 Vol. 36 | pages 123 à 136

ISSN 2262-5305

ISBN 9782747228053

Article disponible en ligne à l'adresse :

https://www.cairn.info/revue-journal-de-gestion-et-d-economiemedicales-2018-2-page-123.htm

Distribution électronique Cairn.info pour ESKA.

(C) ESKA. Tous droits réservés pour tous pays.

La reproduction ou représentation de cet article, notamment par photocopie, n'est autorisée que dans les limites des conditions générales d'utilisation du site ou, le cas échéant, des conditions générales de la licence souscrite par votre établissement. Toute autre reproduction ou représentation, en tout ou partie, sous quelque forme et de quelque manière que ce soit, est interdite sauf accord préalable et écrit de l'éditeur, en dehors des cas prévus par la législation en vigueur en France. Il est précisé que son stockage dans une base de données est également interdit. 


\title{
Contrôle de cohérence actes-dispositifs médicaux
}

\section{Consistency check between medical procedures and medical devices}

\author{
Slimane ToubaL
}

Département d'Information Médicale, CHU de Montpellier, Montpellier

Adrien Poreaux

Département d'Information Médicale, CHU de Montpellier, Montpellier

Mathieu Morell

Département d'Information Médicale, CHU de Montpellier, Montpellier

Philippe MaILHE

Département d'Information Médicale, CHU de Montpellier, Montpellier

$$
\text { Jérôme PERREY }
$$

Département : Pharmacie clinique, Dispensation et Economie de Santé. Equipe Dispositifs Médicaux Stériles

Pierre Aubas

Département d'Information Médicale, CHU de Montpellier, Montpellier

$$
\text { Grégoire MERCIER }
$$

Département d'Information Médicale, CHU de Montpellier, Montpellier CEPEL UMR 5112, Université de Montpellier, Montpellier 


\section{RÉSUMÉ}

Introduction : Le codage des actes médicaux doit être optimal d'autant plus qu'il s'agit de pose de dispositifs médicaux implantables (DMI).Un hôpital universitaire a conçu un contrôle (COHERENCE) permettant de vérifier la cohérence entre les données d'actes de pose de DMI et les données de traçabilité. L'objectif de ce travail est d'évaluer l'impact de ce contrôle : bilan quantitatif sur les séjours concernés, revalorisation des séjours contrôlés et des DMI en sus du GHS.

Méthodes : COHERENCE croise les données de codage des actes avec les données de traçabilité des DMI et repère automatiquement les séjours à contrôler. Ces séjours seront traités par le DIM pour vérification de la saisie des actes. En cas de problème de traçabilité, ces séjours seront traités par la Pharmacie.

Résultats : Pour le premier trimestre 2017, 151 séjours repérés par COHERENCE ont nécessité un contrôle. 48\% des contrôles portaient sur les DMI cardio-vasculaires ; 36\% sur les DMI orthopédiques. $38 \%$ des séjours ont nécessité unecorrection d'acte contre $19 \%$ qui relevaient d'un problème de traçabilité. La revalorisation des séjours contrôlés est estimée à $+\mathbf{2 6 1} 667 €$. La part de revalorisation considérée comme imputable à l'action de correction d'actes est estimée à $+\mathbf{1 1 9 0 1 9} €(+4104 €$ /séjour). La rectification de la traçabilité par la Pharmacie a permis une revalorisation des DMI facturés en sus du GHS de +28 $158 €$.

Conclusion : Les résultats obtenus, mettent en évidence l'intérêt financier et sanitaire de l'utilisation d'un programme automatique de contrôle de cohérence actes-dispositifs médicaux.

Mots-clés : Actes médicaux, dispositifs médicaux implantables, revalorisation, traçabilité.

\section{ABSTRACT}

Introduction: Coding medical procedures should be optimal especially concerning Implantable Medical Devices (IMD). A University Hospital conceived a consistency check (COHERENCE) between medical acting data of implanting IMD and traceability data. The aim of this work is to assess the impact of this checking: quantitative review of controlled hospital discharge records, income gain of controlling these records and IMD reimbursed as "add-on list".

Methods: COHERENCE matches data of coding medical procedures with IMD traceability data, generating automatically discharge records to check. These records would be controlled by the DIM to check data entry of medical procedures. In case of error on traceability, Pharmacy would check these records.

Results: For the first 2017 trimester, COHERENCE detected 151 discharge records requiring a control. $48 \%$ of these controls concerned cardiovascular IMD; 36\% Orthopedic IMD. 38\% of these records required a correction on Medical procedure entry versus $19 \%$ with traceability problems. Income gain of the controlled discharge records is estimated at $+261667 €$. The gain of correcting Medical procedure entry was assessed at $+119019 €(+4104 € /$ record). 
Regarding IMD reimbursed as "add-on list”, traceability correction by the Pharmacy permeated an income gain of $+28158 €$.

Conclusion: Results obtained reveal the financial and sanitary impact of using an automatic consistency check between medical procedure and medical devices.

Keywords: Medical procedures, implantable medical devices, income gain, traceability.

\section{INTRODUCTION}

Depuis la parution de l'arrêté du 20 septembre 1994 [1] et de la circulaire du 10 mai 1995 [2], les établissements de soins sont tenus de transmettre leurs données aux services de l'Etat et à l'Assurance maladie. C'est dans ce contexte que le Programme de Médicalisation des Systèmes d'Information (PMSI) a dû être mis en place.Il s'agit d'un programme permettant aux établissements de soins de mesurer leur production médicale grâce à des informations quantifiées et standardisées sur leur activité [3].

Avec la mise en place de la Tarification à l'Activité (T2A) [4], le PMSI est devenu un outil indispensable permettant l'analyse de l'activité médicale des établissements de santé publics et privés et la transmission aux services de l'état et à l'assurance maladie des informations relatives à leurs moyens de fonctionnement et à leur activité $[5,6]$.

La quantification de l'activité médicale se fait grâce au codage des actes et des diagnostics (diagnostic principal ou relié, diagnostics associés significatifs).

$\mathrm{Au}$ centre universitaire hospitalo-universitaire (CHU) concerné, l'activité de codage est confiée aux cliniciens [7]. Ce système de codage, dit " décentralisé », responsabilise les praticiens et leur offre la possibilité de participer à la valorisation financière de leur activité. Néanmoins, dans un souci d'optimisation, un contrôle est réalisé par l'équipe de Techniciens d'Information Médicale (TIM) afin de vérifier et éventuellement de corriger les erreurs de saisie.

Au-delà de l'intérêt financier que peut apporter le codage optimal des actes en termes de revalorisation des séjours, la qualité de l'information saisie est également importante d'autant plus qu'il s'agit d'actes de pose de dispositifs médicaux implantables (DMI).

La traçabilité sanitaire des DMI est une obligation réglementaire $[8,9]$. Son exhaustivité garantit la sécurité des patients et le remboursement des DMI en sus du Groupe Homogène de Séjours (GHS) par la sécurité sociale.

C'est dans ce contexte qu'il a été décidé de réaliser un contrôle permettant de vérifier la cohérence entre les données d'actes de pose de DMI recueillies par le Département d'Information Médicale (DIM) et les données de traçabilité des DMI recueillies par la Pharmacie. Ce contrôle appelé « cohérence Actes/DMI » a été mis en place en 2012 au centre universitaire étudié [10]. Ils' agit d'un programme informatique qui vérifie que pour chaque acte de pose de DMI saisi, il y a au moins un DMI tracé en relation avec le type d'acte. Par exemple, la saisie d'un acte de pose de prothèse totale de coude, nécessite la présence d'au moins un DMI de prothèse 
de coude (tige radiale ou tige humérale) pour satisfaire le contrôle.

Cependant le contrôle mis en place en 2012, présente la principale limite de ne pas permettre de détecter le cas des prothèses composées de plusieurs DMI dont la traçabilité d'un dispositif n'aurait pas été réalisée. Prenons le cas d'une prothèse totale de coude pour laquelle la traçabilité d'une tige radiale est réalisée mais celle de la tige humérale est manquante. Le contrôle de cohérence se satisfait par la seule présence de la tige radiale.

De ce fait, un nouveau programme informatique permettant un contrôle exhaustif de la cohérence actes-DMI, a été conçu. Ce contrôle, intitulé COHERENCE, a pour but d'une part d'optimiser le codage et d'autre part d'obtenir une traçabilité exhaustive des DMI.

L'objectif de ce travail est d'évaluer l'impact de l'utilisation de COHERENCE : bilan quantitatif sur les séjours concernés, revalorisation des séjours contrôlés et des DMI en sus du GHS.

\section{MOYENS ET MÉTHODES}

\section{Repérage des séjours à contrôler}

Pour chaque séjour, le programme COHERENCE récupère d'une part les données relatives aux actes CCAM (Classification Commune des Actes médicaux) saisis, à partir des données extraites du logiciel de prescription DxCare ${ }^{\circledR}$; et d'autres part Les DMI tracés à partir du logiciel de traçabilité PROTHESE®.
Le croisement de ces données se fait via le logiciel SAS $®$ (StatisticalAnalysis System). Le but de ce croisement est de déterminer si les données issues de la traçabilité des DMI sont cohérentes avec celles des actes saisis.

\section{Paramétrage des combinaisons actes-DMI}

Un algorithme définit pour chaque acte CCAM les différentes combinaisons des DMI requis correspondant à différentes façons d'assembler les DMI d'un même montage. Prenons comme exemple l'acte de pose de prothèse totale de coude MFKA003 (Remplacement de l'articulation du coude par prothèse totale, par abord direct). Deux combinaisons de DMI sont paramétrées : « Tige cubitale + Tige humérale + Condyle ou Charnière » ou « Tige cubitale + Tige humérale charnière incluse » (Figure 1).

12 contrôles, correspondant à plusieurs types de DMI, ont été paramétrés. 4 contrôles pour les DMI de chirurgie orthopédique (prothèses d'épaule, de genou, de hanche et de coude), 3 contrôles pour les DMI cardio-vasculaires (stents/emboles, stimulateurs/défibrillateurs et valves cardiaques), et d'autres contrôles pour les neurostimulateurs, les implants cochléaires, les implants mammaires, les prothèses digestifs/pulmonaires, et les DMI d'urologie.

\section{Croisement des données}

Une extraction des données est réalisée toutes les nuits. COHERENCE parcourt pour chaque séjour la liste des actes codés et vérifie qu'il existe au moins une combinaison de DMI présente au sein des DMI 
Figure 1 : Exemple de combinaisons de DMI correspondants aux actes de pose de prothèses au niveau du coude

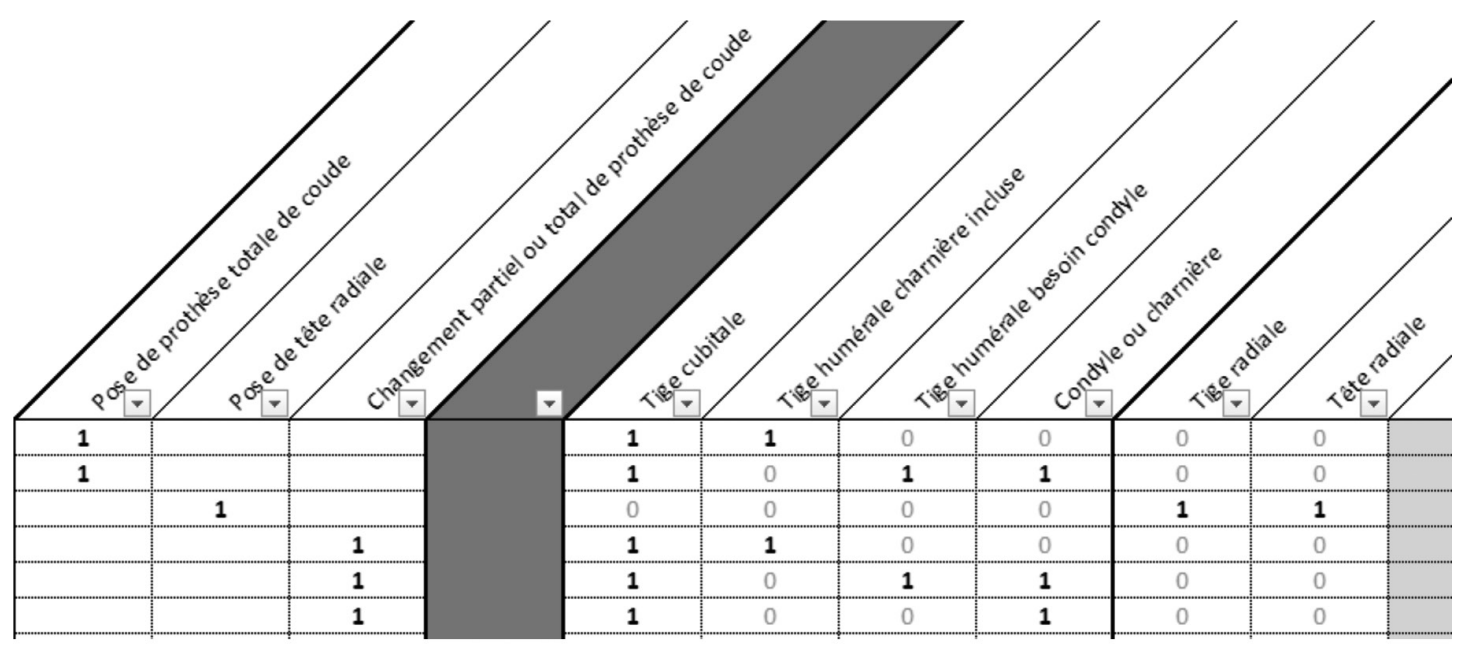

tracés. Si c'est le cas, le séjour est considéré comme cohérent.

Si un des DMI n'est pas tracé, un message d'erreur apparaît : «DMI manquant ». Si, par contre, un DMI est tracé en plus, le séjour apparaît en erreur avec le message : « Acte saturé ».

Par ailleurs, si pour un séjour donné, COHERENCE détecte des DMI tracés mais aucun acte CCAM saisi, le séjour est en erreur avec le message : « Acte manquant».

Les séjours avec un message d'erreur seront traités par l'équipe des TIM et/ou la Pharmacie.

\section{Traitement des séjours à contrôler}

\section{Traitement des séjours par l'équipe des TIM}

Dans un premier temps, les séjours en erreur sont présentés à l'équipe des TIM pour vérification des actes. Une alerte «Cohérence : Acte-DMI» apparait sur l'outil de contrôle qualité conçu au DIM (CQDIM). Cette alerte bloque la facturation du dossier.

Les TIM parcourent les comptes-rendus opératoires (CRO) pour vérifier la saisie des actes chirurgicaux nécessitant la pose d'un DMI. Si la saisie de l'acte (type et nombre) correspond à ce qui est décrit dans les CRO, les TIM valident le dossier. Dans le cas contraire, les TIM procèdent à la modification de l'acte (type et/ou nombre) puis valident le séjour. Une fonction d'aide sur CQDIM propose aux TIM une liste d'actes susceptibles de correspondre à la combinaison de DMI tracés.

Si l'erreur provient de la saisie de l'acte, la correction par les TIM lève l'alerte et fait passer le dossier en facturation. Dans le cas contraire, le dossier reste en alerte et sera transmis à l'interne en Pharmacie du DIM. 
Traitement des séjours par l'interne en Pharmacie du DIM

L'interne en Pharmacie du DIM traite les séjours pour lesquels une erreur persiste malgré un contrôle par les TIM. Il accède à une liste de séjours à contrôler sur l'outil CQDIM. Chaque séjour apparait avec un type d'erreur : « Acte manquant », «DMI manquant » ou « Acte saturé ».

Si l'erreur est de type «Acte manquant », l'interne s'assure d'abord, par la lecture du dossier médical, de l'absence d'un acte chirurgical nécessitant la pose du ou des DMI tracés. Si aucun n'acte n'a été réalisé, le séjour est envoyé à la Pharmacie pour vérification de la saisie de la traçabilité.

Si l'erreur est de type «DMI manquant », l'interne parcourt le CRO pour récupérer des informations sur les DMI posés (type, référence, numéro de lot, numéro de série...) puis transmet le dossier à la Pharmacie pour compléter la traçabilité.

Si l'erreur est de type " Acte saturé », l'interne parcourt le CRO pour s'assurer de la cohérence entre les DMI réellement posés et la saisie de la traçabilité. Si un ou plusieurs DMI sont tracés en plus, il transmet le dossier à la Pharmacie pour rectification.

L'interne du DIM peut être amené à faire des modifications dans le paramétrage du programme. Il alimente la base de données en intégrant les DMI nouvellement référencés.

\section{Traitement des séjours par la Pharmacie}

La Pharmacie traite les séjours pour lesquels une erreur de traçabilité a été détectée après contrôle par les TIM et l'interne du DIM. Le pharmacien accède à la liste des séjours à contrôler sur l'outil CQDIM.

Il vérifie la saisie de la traçabilité sur PROTHESE $®$ à partir des bons de traçabilité déjà envoyés par le bloc opératoire. Il récupère les bons manquants, dans le cas échéant, et réalise les modifications nécessaires (rajout, suppression, rectification).

\section{Méthode d'analyse de l'impact financier des contrôles de cohérence « Actes-DMI »}

Afin d'apprécier l'impact financier du contrôle de cohérence, nous avons réalisé une extraction des données des séjours à partir de l'outil CQDIM.

Entre le $1^{\text {er }}$ janvier et le 31 mars 2017 , tous les séjours concernés par la pose de DMI ont été recensés. Nous distinguons les séjours validés automatiquement par le programme de ceux qui ont présenté une alerte «Cohérence : Acte-DMI». Les séjours validés après contrôle par les TIM et/ou la Pharmacie sont recensés et différenciés selon les actions réalisées (correction acte, correction traçabilité, paramétrage, validation forcée).

L'impact financier du contrôle de cohérence a été estimé grâce à la revalorisation des séjours et des DMI en sus du GHS.

La revalorisation des séjours contrôlés a été estimée en calculant l'écart de valorisation des GHS avant et après contrôle. Nous avons mis en évidence les séjours valorisés après correction d'acte par les TIM et ayant conduit à une modification de la racine du GHM sans modification du diagnostic principal (DP). 
La revalorisation des DMI en sus du GHS a été estimée également en calculant l'écart du montant des DMI facturés en sus du GHS avant et après rectification de la traçabilité par la Pharmacie.

Les variables continues ont été décrites par leur moyenne et leur écart-type ; les variables qualitatives par les effectifs et les pourcentages. Les différents écarts estimés ont été soumis au test de McNemar avec un seuil de significativité statistique (p-value) fixé à 0.05 .

\section{RÉSULTATS}

\section{Bilan quantitatif des séjours contrôlés}

Entre le $1^{\text {er }}$ janvier et le 31 mars 2017, 1395 séjours concernés par la pose d'un DMI ont été validés. 1244 séjours (89\%) ont été validés automatiquement par le programme contre 151 séjours (11\%) ayant nécessité un contrôle par le DIM et/ou la Pharmacie.

72 séjours contrôlés (48\%) portaient sur la pose de DMI en cardio-vasculaire (stents/emboles, stimulateurs/défibrillateurs et valves cardiaques) ; 55 séjours (36\%) sur la pose de DMI en chirurgie orthopédique (coude, épaule, hanche et genou) et 11 séjours $(7 \%)$ concernaient la pose de neurostimulateurs. Le reste de séjours est réparti entre les autres types de DMI (cf. figure 2).

Parmi les séjours contrôlés, 57 séjours (38\%) ont nécessité une correction d'acte contre 27 séjours (19\%) qui relevaient d'un problème de traçabilité. Le reste des séjours a conduit soit à une amélioration du paramétrage $(13 \%)$ soit à une validation forcée $(30 \%)$ du fait d'un problème sur le logiciel de saisie des actes ou du fait des limites du programme (cf. figure 3 ).

$61 \%$ des corrections d'acte ont été réalisées sur les séjours avec pose de DMI

Figure 2 : Proportion des séjours contrôlés par type de DMI

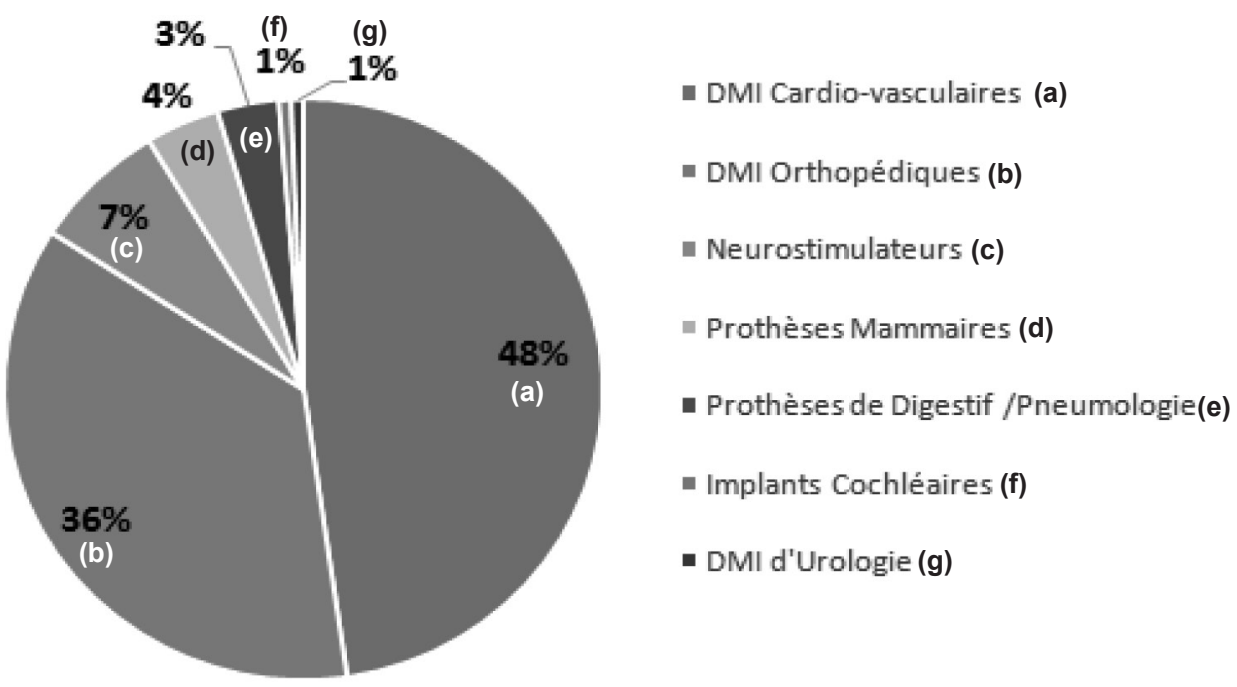


Figure 3 : Proportion des séjours contrôlés par type d'action correctrice

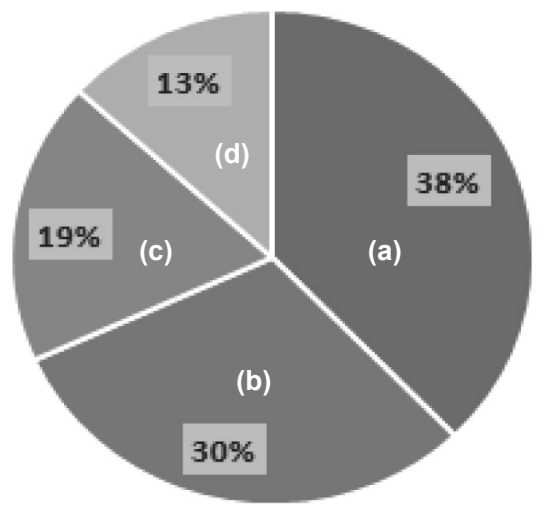

- Correction d'acte (a)

- Validation forcée (b)

- Rectification de traçabilité (c)

- Paramétrage (d)

en cardio-vasculaire principalement sur la pose de stent ou les actes d'embolisation (35\%). $28 \%$ des corrections d'acte ont porté sur les DMI de chirurgie orthopédique avec $19 \%$ pour les DMI de prothèse de hanche.

$59 \%$ des corrections de traçabilité ont porté sur les DMI de chirurgie orthopédique ( $30 \%$ pour les DMI de hanche et $22 \%$ pour le genou). $19 \%$ des corrections de traçabilité ont concerné les DMI de cardio-vasculaire ( $15 \%$ pour les stents ou les emboles). $19 \%$ des corrections de traçabilité ont concerné des DMI de prothèses mammaires.

\section{Revalorisation des séjours contrôlés}

Les 151 séjours contrôlés ont été

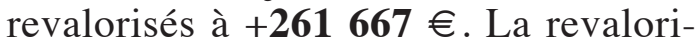
sation des séjours n'étant pas forcément directement imputable au seul contrôle de cohérence, nous avons estimé la part des séjours avec seulement une correction d'actes à $\mathbf{+ 1 4 5 3 1 2} €$. Parmi ces séjours, 36 séjours ont subi une correction d'actes ayant conduit à un changement de racine de GHM. La revalorisation de ces séjours a été estimée à $+\mathbf{1 3 0} 753 €$ soit $+\mathbf{3} 632 €$ par séjour.

Le changement de racine GHM est dû à un changement d'acte et/ou un changement de diagnostic principal (DP). La revalorisation des séjours pour lesquels il y a eu un changement de racine de GHM suite à une correction d'acte de pose de DMI sans changement de DP est estimée à $+\mathbf{1 1 9 0 1 9} €$ soit $\mathbf{+ 4 1 0 4} €$ par séjour (cf. tableau I). Cette revalorisation peut être considérée comme imputable à l'action de correction d'acte par les TIM suite au contrôle de cohérence.

L'analyse des séjours ayant eu un changement de racine GHM sans changement de DP montre qu'avant contrôle $82 \%$ des séjours étaient rattachés à la racine $\mathrm{M}$. Après contrôle les racines $\mathrm{C}$ et $\mathrm{K}$ représentent la plus grande proportion des séjours avec $52 \%$ et $45 \%$ respectivement (cf. tableau I).

Pour exemple, le passage d'un séjour du GHM 05K103 « Actes diagnostiques par voie vasculaire » au GHM 05C193 "Poses d'un défibrillateur cardiaque » est à l'origine d'une revalorisation de 
Tableau I : Récapitulatif de la valorisation des séjours concernés par le contrôle de cohérence

\begin{tabular}{|l|r|r|r|r|}
\cline { 2 - 4 } \multicolumn{1}{c|}{} & Avant contrôle & Après contrôle & \multicolumn{1}{c|}{ Ecart } & p-value*** \\
\hline Valorisation GHS* & $78490 €$ & $197521 €$ & $+119031 €$ & \multirow{2}{*}{$<0.001$} \\
\hline Valorisation GHS par séjour* & $2707 €( \pm 1563)$ & $6811 €( \pm 4510)$ & $+4104 €$ & \\
\hline Valorisation DMI** & $9194 €$ & $37352 €$ & $+28158 €$ & \multirow{2}{*}{0.002} \\
\hline Valorisation DMI par séjour** & $400 €( \pm 1479)$ & $1624 €( \pm 4209)$ & $+1224 €$ & \\
\hline Nombre de DMI facturés & 23 & 63 & +40 & \\
\hline Valorisation totale & $87684 €$ & $234873 €$ & $+147189 €$ & \multirow{2}{*}{$<0.001$} \\
\hline Valorisation totale par séjour & $1686 €( \pm 1672)$ & $4517 €( \pm 4355)$ & $+2831 €$ & \\
\hline Proportion de M* & $86 \%$ & $3 \%$ & $-83 \%$ & $\mathrm{M}:<0.001$ \\
Proportion de C* & $7 \%$ & $52 \%$ & $+45 \%$ & $\mathrm{C}: 0.002$ \\
Proportion de K* & $7 \%$ & $45 \%$ & $+38 \%$ & $\mathrm{~K}: 0.006$ \\
\hline
\end{tabular}

*séjours avec un changement de racine de GHM suite à une correction d'acte de pose de DMI sans changement de DP

**séjours avec une rectification de la traçabilité par la Pharmacie

***test de McNemar avec un seuil de significativité statistique (p-value) fixé à 0.05

$\mathbf{+ 1 6} 721 €$. Le passage du GHM 05M111 "Cardiopathies congénitales et valvulopathies, âge supérieur à 17 ans » au GHM 05K211 «Poses de bioprothèses de valves cardiaques par voie vasculaire » est à l'origine d'un gain de valorisation de $+27794 €$ pour sept séjours contrôlés (cf. tableau II).

\section{Revalorisation des DMI en sus du GHS}

L'écart du montant des DMI facturés en sus du GHS entre avant et après contrôle des séjours pour lesquels il y a eu un problème de traçabilité est estimé à $+\mathbf{5 8 6 3 7} €$ pour 51 DMI. Les DMI des séjours pour lesquels il y a eu une rectification sur la traçabilité de la part de la Pharmacie, ont été revalorisés à $+\mathbf{2 8} \mathbf{1 5 8} €$. Les DMI les plus concernés par les problèmes de traçabilité sont les implants d'orthopédie (59\%), les prothèses mammaires (18\%) et les implants de cardio-vasculaire (19\%) (cf. figure 4).

\section{DISCUSSION}

Dans un objectif d'optimisation du codage et d'exhaustivité de la traçabilité des DMI, un programme informatique appelé COHERENCE a été conçu par le DIM du CHU concerné. Il permet de contrôler la cohérence entres les actes saisis et la traçabilité des DMI.

Pour le trimestre de Janvier à Mars 2017, 1244 séjours sur les 1395 séjours concernés par la pose de DMI ont été contrôlés et validés automatiquement par 
Tableau II : Revalorisation des séjours contrôlés avec une correction d'acte de pose de DMI ayant pour conséquence un changement de racine de GHM sans changement de DP ( $\mathrm{N}$ : nombre de séjours)

\begin{tabular}{|c|c|c|c|c|}
\hline Contrôle & $\mathbf{N}$ & Racine GHM avant contrôle & Racine GHM après contrôle & Ecart \\
\hline Epaule & 2 & $\begin{array}{l}\text { Maladies osseuses et arthropathies } \\
\text { spécifiques }\end{array}$ & Prothèses d'épaule & $+5264 €$ \\
\hline Genou & 1 & $\begin{array}{l}\text { Maladies osseuses et arthropathies } \\
\text { spécifiques }\end{array}$ & Prothèses de genou & $+4070 €$ \\
\hline \multirow{4}{*}{ Hanche } & 1 & $\begin{array}{l}\text { Maladies osseuses et arthropathies } \\
\text { spécifiques }\end{array}$ & $\begin{array}{l}\text { Interventions pour reprise de } \\
\text { prothèses articulaires }\end{array}$ & $+6696 €$ \\
\hline & 3 & $\begin{array}{l}\text { Fractures de la hanche et du } \\
\text { bassin }\end{array}$ & $\begin{array}{l}\text { Prothèses de hanche pour } \\
\text { traumatismes récents }\end{array}$ & $+7613 €$ \\
\hline & 1 & $\begin{array}{l}\text { Interventions sur la hanche et le } \\
\text { fémur sauf traumatismes récents, } \\
\text { âge supérieur à } 17 \text { ans }\end{array}$ & $\begin{array}{l}\text { Interventions pour reprise de } \\
\text { prothèses articulaires }\end{array}$ & $+1209 €$ \\
\hline & 1 & $\begin{array}{l}\text { Suites de traitement après } \\
\text { une affection de l'appareil } \\
\text { musculosquelettique ou du tissu } \\
\text { conjonctif }\end{array}$ & $\begin{array}{l}\text { Interventions pour reprise de } \\
\text { prothèses articulaires }\end{array}$ & $+5031 €$ \\
\hline $\begin{array}{l}\text { Neuro- } \\
\text { stimulateurs }\end{array}$ & 1 & $\begin{array}{l}\text { Explorations et surveillance pour } \\
\text { affections du système nerveux }\end{array}$ & Pose d'un stimulateur cérébral & $+6775 €$ \\
\hline \multirow{6}{*}{$\begin{array}{l}\text { Stents } \\
\text { OuEmboles }\end{array}$} & 1 & $\begin{array}{l}\text { Autres lésions traumatiques } \\
\text { intracrâniennes, sauf commotions }\end{array}$ & $\begin{array}{l}\text { Craniotomies pour traumatisme, } \\
\text { âge supérieur à } 17 \text { ans }\end{array}$ & $+10455 €$ \\
\hline & 2 & Infarctus aigu du myocarde & $\begin{array}{l}\text { Endoprothèses vasculaires avec } \\
\text { infarctus du myocarde }\end{array}$ & $+2566 €$ \\
\hline & 2 & $\begin{array}{l}\text { Troubles vasculaires } \\
\text { périphériques }\end{array}$ & $\begin{array}{l}\text { Endoprothèses vasculaires sans } \\
\text { infarctus du myocarde }\end{array}$ & $+1496 €$ \\
\hline & 1 & $\begin{array}{l}\text { Troubles vasculaires } \\
\text { périphériques }\end{array}$ & $\begin{array}{l}\text { Chirurgie majeure de } \\
\text { revascularisation }\end{array}$ & $+3291 €$ \\
\hline & 1 & $\begin{array}{l}\text { Cardiopathies congénitales et } \\
\text { valvulopathies, âge supérieur } \\
\text { à } 17 \text { ans }\end{array}$ & $\begin{array}{l}\text { Poses de bioprothèses de valves } \\
\text { cardiaques par voie vasculaire }\end{array}$ & $+4587 €$ \\
\hline & 1 & $\begin{array}{l}\text { Actes thérapeutiques sur les } \\
\text { artères par voie vasculaire, âge } \\
\text { supérieur à } 17 \text { ans }\end{array}$ & $\begin{array}{l}\text { Endoprothèses vasculaires sans } \\
\text { infarctus du myocarde }\end{array}$ & $-265 €$ \\
\hline
\end{tabular}




\section{Tableau II (Suite)}

\begin{tabular}{|c|c|c|c|c|}
\hline Contrôle & $\mathbf{N}$ & Racine GHM avant contrôle & Racine GHM après contrôle & Ecart \\
\hline \multirow{4}{*}{$\begin{array}{l}\text { Stimulateurs } \\
\text { Défibrillateurs }\end{array}$} & 1 & Syncopes et lipothymies & $\begin{array}{l}\text { Poses d'un stimulateur cardiaque } \\
\text { permanent sans infarctus aigu } \\
\text { du myocarde, ni insuffisance } \\
\text { cardiaque congestive, ni état de } \\
\text { choc }\end{array}$ & $+1629 €$ \\
\hline & 1 & $\begin{array}{l}\text { Actes diagnostiques par voie } \\
\text { vasculaire }\end{array}$ & $\begin{array}{l}\text { Poses d'un défibrillateur } \\
\text { cardiaque }\end{array}$ & $+16721 €$ \\
\hline & 1 & $\begin{array}{l}\text { Autres affections de l'appareil } \\
\text { circulatoire }\end{array}$ & $\begin{array}{l}\text { Poses d'un défibrillateur } \\
\text { cardiaque }\end{array}$ & $+14547 €$ \\
\hline & 1 & $\begin{array}{l}\text { Poses d'un stimulateur cardiaque } \\
\text { permanent avec infarctus aigu } \\
\text { du myocarde ou insuffisance } \\
\text { cardiaque congestive ou état } \\
\text { de choc }\end{array}$ & $\begin{array}{l}\text { Insuffisances cardiaques et états } \\
\text { de choc circulatoire }\end{array}$ & $+1540 €$ \\
\hline Valves & 7 & $\begin{array}{l}\text { Cardiopathies congénitales et } \\
\text { valvulopathies, âge supérieur } \\
\text { à } 17 \text { ans }\end{array}$ & $\begin{array}{l}\text { Poses de bioprothèses de valves } \\
\text { cardiaques par voie vasculaire }\end{array}$ & $+27794 €$ \\
\hline
\end{tabular}

Figure 4 : Proportion des séjours avec problèmes de traçabilité

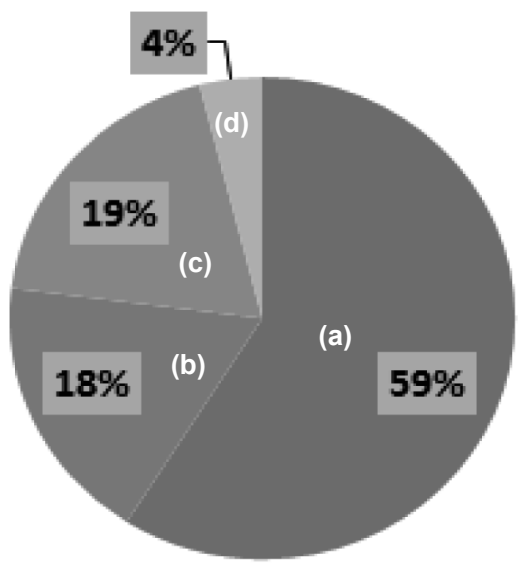

DMI Orthopédiques (a)

- Prothèses Mammaires (b)

- DMI Cardio-vasculaires (c)

Neurostimulateurs (d) 
COHERENCE. Le programme permet donc de réduire considérablement le nombre de séjours à traiter par l'équipe du DIM et/ou par la Pharmacie. Ainsi seuls 151 séjours $(11 \%)$ ont nécessité un contrôle manuel humain. De plus, une fonction d'aide sur CQDIM propose aux TIM et aux pharmaciens une liste d'actes et/ou de DMI susceptibles de résoudre le problème.

57 séjours $(38 \%)$ ont nécessité une correction d'acte. Ceci pourrait s'expliquer par la complexité de la classification CCAM qui, pour des impératifs d'exhaustivité, est riche d'un nombre très important d'actes parfois proches les uns des autres rendant ainsi le travail de saisie par le clinicien complexe et très chronophage [11]. 61\% des séjours ayant nécessité une correction d'acte concernent les séjours avec pose de DMI en cardio-vasculaire. Ceci pourrait s'expliquer par la richesse et la complexité des différents actes en lien avec la pose d'un dispositif en cardio-vasculaire. En effet, un peu plus de la moitié des actes de compatibilité CCAM et groupes de DM décrits par l'ATIH (Agence technique de l'information sur l'hospitalisation) correspond à des actes de pose de DMI en cardio-vasculaire [12].

La correction des actes par les TIM a eu un impact financier considérable. En effet, la revalorisation des séjours ayant eu un changement de racine GHM suite à une correction d'acte sans changement de DP a été estimée à $+119019 €$ soit $+4104 €$ par séjour. Cette revalorisation est en lien direct avec les alertes CQDIM générées par le programme COHERENCE. En l'absence d'alerte «Cohérence Acte-DMI », les TIM n'auraient pas contrôlé la saisie des actes en lien avec la pose de DMI et n'auraient donc pas corrigé ces derniers afin qu'ils soient fidèles au compte rendu opératoire. Ceci exige un travail supplémentaire de la part des TIM, mais non moins gratifiant. Une nouvelle compétence d'analyse des CRO en lien avec la pose de DMI vient enrichir la fonction des TIM de plus en plus professionnalisante [13].

27 séjours (19\%) ont nécessité une rectification de la traçabilité. La majorité de ces séjours $(59 \%)$ concernent la pose de DMI en chirurgie orthopédique. Différentes causes inhérentes au circuit du DMI dans le CHU concerné peuvent expliquer ces problèmes de traçabilité. Nous pouvons citer : la saisie manuelle des numéros de lots et des références des dispositifs [14], la perte des bons de traçabilité, le retard dans la mise à disposition des bons de traçabilité, etc. La mise en place d'une traçabilité automatique par simple lecture scanner permettrait de s'affranchir d'une grosse partie de ces erreurs [15].

Les problèmes de traçabilité ont concerné principalement les DMI de chirurgie orthopédique $(59 \%)$, les prothèses mammaires (18\%) ainsi que les DMI de cardio-vasculaire (19\%). La chirurgie orthopédique se caractérise par la complexité des différents montages prothétiques, ce qui explique en partie les problèmes de traçabilité rencontrés.

Concernant les séjours pour lesquels une rectification de la traçabilité a été opérée à la suite de l'alerte « cohérence actes-DMI », la revalorisation des DMI facturés en sus du GHS a été estimée à $+28158 €$. Ces chiffres témoignent du rôle important du DIM dans la détection des erreurs de traçabilité et renforce le lien DIM-Pharmacie. Cette collaboration est capitale, tant sur le plan sanitaire que financier.

Comme tout outil informatique, le programme COHERENCE présente des limites. $44 \%$ des séjours en alerte 
« cohérence actes-DMI » sont considérés comme faux positifs. $13 \%$ ont permis de déceler les points faibles du programme et ont abouti à un changement de paramétrage afin de traiter les combinaisons actes/DMI les plus pertinentes.

$30 \%$ des alertes étaient liées soit à un problème dans le logiciel de saisie des actes soit à un problème lié à l'algorithme paramétré. En effet, si plusieurs actes de pose de DMI sont saisis pour un même séjour (exemple : pose de prothèse de genou et pose de prothèse de hanche) ; COHERENCE n'est pas en mesure de distinguer quel DMI attribuer à quel acte. Le programme considère tous les DMI comme faisant partie du premier acte, il en résulte un message d'erreur avec « acte saturé » pour le premier acte et «DMI maquant» pour le deuxième acte.Une plus grande période d'évaluation permettrait d'identifier tous ces faux positifs et d'améliorer ainsi la programmation de COHERENCE.

Dans une étude réalisée dans le cadre d'une thèse d'exercice, COHERENCE a montré une sensibilité et une spécificité supérieures à 95\% dans le traitement des séjours de pose de prothèse de hanche[16]. Il serait intéressant de déterminer les performances de COHERENCE pour le traitement des autres contrôles, notamment celui des DMI en cardio-vasculaire.

Les résultats obtenus pendant le premier trimestre 2017, mettent en évidence l'intérêt financier et sanitaire de l'utilisation d'un programme automatique de contrôle de cohérence entre les actes saisis et la traçabilité des DMI. Cet outil permettrait, d'une part une revalorisation des séjours concernés par une implantation d'un dispositif médical, et d'autre part une traçabilité exhaustive des DMI.
Par ailleurs, ce programme renforce les liens professionnels entre le DIM et la Pharmacie.

\section{BIBLIOGRAPHIE}

[1] Arrêté du 20 septembre 1994 relatif au recueil et au traitement des données d'activité médicale et de coût. Code de la santé publique[En ligne]. Disponible : https://www.legifrance.gouv.fr/affichTexte. do?cidTexte=JORFTEXT000000348993

[2] Circulaire DH/DSS $/ 95 / \mathrm{N}^{\circ} 23 \mathrm{du} 10 \mathrm{mai}$ 1995 relative à la généralisation du dispositif technique, expérimenté en région LanguedocRoussillon, demesure de l'activité et des coûts des établissements hospitaliers sous compétence tarifaire de l'état, à l'aide des informations provenant du système d'information médicalis. ATIH [En ligne]. Disponible:http://www.atih. sante.fr/sites/default/files/public/content/990/ Cir_10-5-95.pdf

[3] Morkos M. L'acronyme au centre de toutes les conversations : le PMSI, qu'est-ce que c'est ? Hospitalia $2009 ; 8 ; 48-50$. Disponible : http:// www.web100t.fr/Presse/2009-10\%20PMSI.pdf

[4] LOI n 2003-1199 du 18 décembre 2003 de financement de la sécurité sociale pour 2004 [En ligne]. Disponible : http://www.legifrance.gouv. fr/eli/loi/2003/12/18/SANX0300139L/jo/texte

[5] Article L.6113-7 relatif à l'évaluation, l'accréditation et l'analyse de l'activité des établissements. Code de la santé publique [En ligne]. Disponible : https://www.legifrance. gouv.fr/affichCodeArticle.do?cidTexte=LEGITEXT000006072665\&idArticle $=$ LEGIARTI000006690710

[6] Article L.6113-8 relatif à l'évaluation, l'accréditation et l'analyse de l'activité des établissements. Code de la santé publique [En ligne]. Disponible : https://www.legifrance. gouv .fr/affichCodeArticle.do?cidTexte=LEGITEXT000006072665\&idArticle $=$ LEGIARTI000006690711

[7] Lehmann M, Aubas P, editors. Le contrôle de qualité du codage des résumés d'unité Médicale au chu de Montpellier : contexte, méthodes, principaux résultats. EMOIS; 2007; Nancy. 
[8] Décret n²006-1497 du 29 novembre 2006 fixant les règles particulières de la matériovigilance exercée sur certains dispositifs médicaux et modifiant le code de la santé publique (Dispositions réglementaires). Code de la santé publique [En ligne]. Disponible : https://www.legifrance.gouv.fr/affichTexte. do? cidTexte $=$ LEGITEXT000006054829

[9] Arrêté du 26 janvier 2007 relatif aux règles particulières de la matériovigilance exercée sur certains dispositifs médicaux, pris en application de l'article L. 5212-3 du code de la santé publique. Code de la santé publique [En ligne]. Disponible : https://www.legifrance.gouv.fr/eli/ arrete/2007/1/26/SANP0720369A/jo

[10] Blanco C, Soulairol I, Selvy N, et al. Dispositifs Médicaux Implantables: Cohérence d'information entre traçabilité sanitaire et codification des actes médicaux. $21^{\text {es }}$ Journées Nationales d'Etudes sur les Dispositifs Médicaux Europharmat Lyon ; 2011.

[11] Frenkiel J, audition : comptes rendus de la mission d'évaluation et de contrôle de la sécurité sociale [En ligne]. Disponible : http://www. senat.fr/compte-rendu-commissions/20120130/ mecss.html
[12] Compatibilité entre actes CCAM et groupes de DM [En ligne]. Disponible : http://www. atih.sante.fr/compatibilite-entre-actes-ccamet-groupes-de-dm

[13] Kunzli b, Deschamps c, Vicente P, et al. Rôle du cadre dans un DIM dans un contexte de professionnalisation du codage : retour d'expérience après 18 mois dans un CHU. Revue d'Épidémiologie et de Santé Publique. 2014 ;62:s87-s8.

[14] Statistique Canada. Lignes directrices concernant la qualité. 2009; 5; 40 [En ligne]. Disponible : http://www.statcan.gc.ca/pub/12-539-x/12539-x2009001- fra.pdf

[15] Durand L, Drouot S, Frontini S, et al. Codification industrielle des DMI : quelles informations reconnues par lecture scanner? Le Pharmacien Hospitalier et Clinicien. 2014; 49: 317-318.

[16] Poreaux A. «Conception et évaluation d'un outil informatique de contrôle de la cohérence entre les actes du PSMI et les dispositifs médicaux implantables », Thèse de docteur en Pharmacie, Montpellier,UFR des Sciences Pharmaceutiques et Biologiques, 2016, 79 p. 